This is an author produced version of a paper published in Canadian Journal of Forest Research.

This paper has been peer-reviewed but may not include the final publisher proof-corrections or pagination.

Citation for the published paper:

Sebastian Schnell, Jonas Wikman, Göran Ståhl. (2013) Relascope sampling for crown ratio estimation. Canadian Journal of Forest Research. Volume: 43, Number: 5, pp 459-468.

http://dx.doi.org/10.1139/cjfr-2012-0230.

Access to the published version may require journal subscription.

Published with permission from: NRC Research Press.

Standard set statement from the publisher:

All authors who publish in NRC Research Press journals may at any time in the publication process self-archive their submitted and/or accepted manuscript on their institution's Web site or in an open-access repository such as PubMed Central (this is sometimes known as "green" open access).

Epsilon Open Archive http://epsilon.slu.se 


\title{
Relascope sampling for crown ratio estimation
}

\author{
Sebastian Schnell, Jonas Wikman, Göran Ståhl \\ Department of Forest Resource Management, Swedish University of Agricultural Sciences, Umeå, Sweden
}

Published 2013 in Canadian Journal of Forest Research), 459-468, dx.doi.org/10.1139/cjfr-2012-0230

\begin{abstract}
In this study we apply vertical angle count sampling to estimate the crown ratio of trees in unthinned forest stands. The rationale is to be able to quickly assess the relative crown size of forest stands to support thinning decisions by simply counting trees. We provide estimators and discuss their precision based on pilot studies in Scots Pine plantations in northern Sweden. A separate study was conducted to investigate the amount of measurement errors, i.e. how many trees are wrongly selected or overseen when using the method. Sampling errors for estimating crown ratio were found to be remarkably low, partly due to high correlation between crown length and tree height and partly due to low variability in the study sites. Measurement errors were in the range of what is commonly obtained with horizontal angle count sampling for basal area estimation.
\end{abstract}

Keywords: Relascope, vertical angle count, crown ratio, Pinus sylvestris

\section{Introduction}

Angle count sampling (Bitterlich 1948; Grosenbaugh 1958) has long been a standard method in forest inventory for basal area estimation. The main advantages are its ease of application and its good precision for estimating basal area and related variables. Trees are selected by projecting an opening angle on a tree's stem at breast height and including the tree into the sample if its stem appears larger than the opening angle. Simple counts without additional measurements are sufficient for obtaining an estimate of basal area per hectare. The method automatically selects trees proportional to their size in terms of basal area. Various modifications of the original idea exist. Hirata (1955) proposed using the opening angle vertically and aiming at tree heights instead of diameters, resulting in estimates of quadratic mean height provided additional stem counts are available. The method however has no direct use in forestry (Bitterlich 1984) because of the squared units. A combination of inventory lines and the angle count method was first proposed by Strand (1957) both for horizontal and vertical opening angles, the latter one providing cylinder volumes that could be used for estimating stand volume if appropriate form factors are known (see Bitterlich 1984, pp 43-46). Direct volume estimation is possible by a method called critical height sampling, first proposed by Kitamura (1962), where the stem of a tree is scanned by an angle gauge until its diameter exactly fits the opening angle. This results in a critical height, which is used in the volume estimators (see Gove et al. 2005). Apart from standing trees, angle count sampling has also been developed for the assessment of lying dead wood. A first description can be found in Ståhl (1998) for sample lines, later complemented by an application for points by Gove et al. (1999). With these methods, angle gauges with large opening angles are used to aim at $\operatorname{logs}$ that lay on the forest floor. Methods for volume estimation of dead wood analogous to critical height sampling are available as well (see Gove et al. 2005; Ståhl et al. 2010). Instead of stem diameters Stenberg et al. (2008) used a relascope to aim at crown diameters to estimate canopy cover.

In this paper we further develop and discuss what Bitterlich (1984, p 41) called vertical angle count sampling and Grosenbaugh (1958) vertical point- or line sampling. Trees are selected based on their height or other vertical features, such as their crown length, and instead of a sum of tree basal areas a sum of heights (lengths) or height squares can be estimated based on simple tree count data. Since the method was first mentioned by Hirata (1955) and Strand (1957) it has not been very much used in practice. To our knowledge, the earliest application is due to Bickerstaff (1961) who used vertical line sampling for determining stocking levels in young stands. Similar studies are the ones by Beers (1974), Hitchcock (1979), and Lappi et al. (1983); all having in common that the method is applied in regeneration stands. In Brown and Mugasha (1988) the use of vertical point sampling in the first stage of a two stage sampling scheme is described. The application scenario is dense stands of small trees where poor visibility makes the common angle count method difficult to apply. Vertical point sampling is used to select a larger than necessary sample of trees. A traditional angle count is then performed using diameter and distance measurements from the previously selected trees. In another study, trees selected by a vertical point sample were counted as competitors to a certain tree (Mugasha 
1989). Lynch (1990) derived stand volume estimators based on tree counts from a vertical line sample. The latest publication on vertical point sampling (Ducey and Kershaw 2011) replaces the relascope with a digital camera to overcome the problem of a "challenging field technique". Apart from applying the relascope principle to other study variables or objects of interest, there is also ongoing research on improving estimators for traditional basal areal estimation (e.g. McTague, 2010).

In this study we combine aggregated height and crown length measures, derived from either vertical point or line sampling, to estimate the crown ratio of a stand. This type of measurements would allow quick assessments of unthinned stands to support thinning decisions. A general rule for Scots pine plantations in Sweden is that a first thinning should take place at dominant heights between $10 \mathrm{~m}$ and $13 \mathrm{~m}$ (Agestam 2009), shortly after crown closure to prevent crowns from getting too small, which would lead to a decrease of the growth potential of trees (e.g. Lessard et al. 2001; Leites et al. 2009). It is thereby envisaged that the crown ratio should not be less than $50 \%$.

Further, measures of crown size (crown length and crown ratio) are commonly used to model tree vigour and wood quality, as well as diameter increment and tree mortality (see e.g. Hasenauer and Monserud, 1996 and references therein). In addition, Tobin et al. (2006) found that the inclusion of crown characteristics in allometric equations improved the estimation of leaf biomass and leaf area index. These examples, however, relate to measures at individual tree level and not to a population ratio as our method provides.

The objective of the study was to develop the theory for crown ratio estimation based on vertical angle count sampling and to conduct initial field tests to assess the performance of the method in terms of sampling and measurement errors. Since the field tests were conducted in a limited number of stands with low between-stand variability, the results only provide some first indications of the feasibility of using this method in practice.

\section{Material \& methods}

\section{Definition of crown ratio}

Given a population of $N$ trees (e.g. within a stand), where for each tree $i$ the two variables crown length $y_{c, i}$ and tree height $y_{h, i}$ are known, a population crown ratio (cf. Cochran 1977, pp 30-35) can be defined as:

$$
\text { [1] } \quad r_{c}=\frac{\frac{1}{N} \sum_{i=1}^{N} y_{c, i}}{\frac{1}{N} \sum_{i=1}^{N} y_{h, i}}=\frac{\tau_{c}}{\tau_{h}}
$$

Here, $\tau_{c}$ is the total of crown lengths in the population and $\tau_{h}$ is the corresponding total of tree heights. The crown ratio $r_{c}$ is the population parameter we wish to estimate using the vertical angle count method.

For reasons that will become clear later on, we also present an alternative definition, $r_{c, a l t}$, of a population crown ratio:

$$
r_{c, a l t}=\frac{\sqrt{\frac{1}{N} \sum_{i=1}^{N} y_{c, i}^{2}}}{\sqrt{\frac{1}{N} \sum_{i=1}^{N} y_{h, i}^{2}}}=\frac{\sqrt{\tau_{c, s q}}}{\sqrt{\tau_{h, s q}}}
$$

Here, $y_{c, i}^{2}$ and $y_{h, i}^{2}$ are squared crown lengths and tree heights, respectively, and consequently $\tau_{c, s q}$ and $\tau_{h, s q}$ now represent sums of squares. Note that $r_{c}$ and $r_{c, \text { alt }}$ are only equal to each other under certain conditions and usually differ to some extent. We elaborate on this in a later section of the article.

\section{Estimation}

From the definitions (Eq. 1 and Eq. 2) it follows that we can estimate the crown ratio through a ratio estimator, based on estimates of the totals $\hat{\tau}_{c}$ and $\hat{\tau}_{h}$ or $\hat{\tau}_{c, s q}$ and $\hat{\tau}_{h, s q}$, i.e.:

$$
\hat{r}_{c}=\frac{\hat{\tau}_{c}}{\hat{\tau}_{h}} \text { and [4] } \quad \hat{r}_{c, \text { alt }}=\frac{\sqrt{\hat{\tau}_{c, s q}}}{\sqrt{\hat{\tau}_{h, s q}}}
$$

Note that these estimators are only approximately unbiased, even when the estimators of $\hat{\tau}_{c}, \hat{\tau}_{h}, \hat{\tau}_{c, s q}$, and $\hat{\tau}_{h, s q}$ are unbiased. Crown ratio estimation is straightforward once we can estimate the population total of crown lengths and tree heights (or the corresponding totals of squares in case the alternative definition is used). Below, we show how this can be performed using the general Horvitz-Thompson estimation framework (Horvitz and Thompson 1952). We base our derivations on the case where one sample location has been randomly selected within a stand and apply the principle of replicated sampling (e.g. Gregoire and Valentine 2008, p. 216) to estimate the total in case of $s=1,2, \ldots, m$ sample locations:

$$
\hat{\tau}=\frac{1}{m} \sum_{s=1}^{m} \hat{\tau}_{s} \text { and [6] } \quad \hat{\tau}_{s q}=\frac{1}{m} \sum_{s=1}^{m} \hat{\tau}_{s q, s}
$$

Here $\hat{\tau}_{s}$ and $\hat{\tau}_{s q, s}$ are estimates at a single sample location, $s$, for either total crown length or total tree height or, accordingly, the totals of squares. Similarly $\hat{\imath}$ and $\hat{\tau}_{s q}$, as well, represent a generic notation including estimation related to crown length and tree height. We now move on to focus on $\hat{\tau}_{s}$ and $\hat{\tau}_{s q, s}$ for which the Horvitz-Thompson estimators are:

$$
\hat{\tau}_{s}=\sum_{i=1}^{n_{s}} \frac{y_{i}}{\pi_{i}} \text { and [8] } \quad \hat{\tau}_{s q, s}=\sum_{i=1}^{n_{s}} \frac{y_{i}^{2}}{\pi_{i}}
$$

Here $y_{i}$ and $y_{i}^{2}$ are the characteristics (crown length or tree height and the corresponding squares when estimating $\tau_{s q, s}$ ) of interest for tree $i$ included in the sample at location $s$, where $n_{s}$ denotes the number of trees selected at this location. The necessary inclusion probabilities $\pi_{i}$ can be derived using the inclusion zone 
concept (e.g. Gregoire and Valentine 2008, pp 210$215)$ illustrated in Fig. 1. Inclusion zones are typically defined uniquely for each object of interest, e.g. a tree, and the object is selected if the sample location falls within its zone. The size of the zone, $a_{i}$, is proportional to the inclusion probability, which is $\pi_{i}=a_{i} / A$, where $A$ is the size of the sample frame for estimating totals or $10000 \mathrm{~m}^{2}$ for estimates on a per-hectare basis. When applying point sampling - see case a) in Fig. 1 the inclusion zones are circular, centred on a tree and of size $\pi \times D_{i}^{2}$ varying with tree height or crown length as shown later on. Here $D_{i}$ denotes the critical distance between observer and tree, at which the tree fits exactly into the opening angle. For line sampling see case b) in Fig. 1 - the shape of the inclusion zone is rectangular and the size is $D_{i} \times L$, where $L$ is the length of the sample line and $D_{i}$ as before the critical distance. Here, the case of a non-centred sample line with measurements to one side, as applied in this study, is shown. The shapes of the two inclusion zones correspond to the ones from horizontal point and line sampling (e.g. Gregoire and Valentine 2008, p 360).

From Eq. 7, Eq. 8 and Fig. 1, we need to be able to determine the sizes of the inclusion zones in order to calculate the needed inclusion probabilities. For both line and point sampling the areas depend on tree height or crown length. The derivation of the inclusion zone's size is illustrated in Fig. 2 using the intercept theorem, which describes the relationship of line segments resulting from two intersecting lines cut by two parallels. Under a) and b) the selection for tree height and crown length in levelled terrain is shown, respectively, while under c) and d) two extreme cases of crown measurement for sloped terrain are illustrated with observer positions (the black dots) either above the tree top or below the stem base. For deriving $D_{i}$, in most of the cases we need to establish two triangles, defined by the two bold and the one dashed line. The bold lines thereby depict the path of rays when observing a tree or a crown through the instrument, which itself is defined by the constants $d$ and either $h_{1}+h_{2}$ for case a) or simply $h_{1}$ for cases b) to d). In some rare cases only one triangle would be needed and that is when the eye of the observer, the lower or upper mark of the instrument, and the tree top, crown or tree base would be on the same horizontal line. The triangle would then be a right angled one with the adjacent side at the observer position. Following this, in all other cases the dashed line is a necessity for calculating $D_{i}$, which must be horizontal to get the correct sizes of the inclusion zones. As a further convention we define $H_{i}=H_{1 i}+H_{2 i}$ and $h=h_{1}+h_{2}$, and make use of the intercept theorem:

$$
\frac{H_{1 i}}{h_{1}}=\frac{D_{i}}{d} \text { and } \frac{H_{2 i}}{h_{2}}=\frac{D_{i}}{d}
$$

For case a) we have $H_{i}=D_{i} \times h_{1} / d+D_{i} \times h_{2} / d$ and rearranging for $D_{i}$ we get the critical distance $D_{i}=$ $H_{i} \times d / h$, which solely depends on the tree height $H_{i}$, as the instrument constants $d$ and $h$ are fixed. The derivation for the other cases in Fig. 1 works in a similar way by solving $H_{i}=H_{1 i}+H_{2 i}$ for $H_{1 i}$, which leads to $D_{i}=H_{1 i} \times d / h_{1}$. In all cases the critical distance can be calculated from tree height or crown length and the instrument constants. As can be seen from cases c) and d) in Fig. 2, the method also works in slopes, as long as the horizontal distance $d$ is kept. Knowing $D_{i}$ the size of the inclusion zones can be calculated. For line sampling the formula is $a_{i}=L \times$ $H_{i} \times d / h$, and for point sampling $a_{i}=\pi \times H_{i}^{2} \times$ $(d / h)^{2}$. Note that when measuring crowns, $H_{i}$ is simply replaced with $H_{1 i}$ from Fig 2, cases b) to d).

From the size of the inclusion zones, it now becomes apparent why we introduced the alternative crown ratio definition in Eq. 2 and its estimator in Eq 4. Applying point sampling, the inclusion zones' size and the corresponding probability depend on either tree height squared or crown length squared. Because of this we have squared units in the denominator of Eq. 8 and as the general aim is to provide estimates without additional measurements, squared units are required in the numerator as well, so that the terms cancel and trees just have to be counted. As a consequence, the alternative crown ratio is connected to the application of point sampling, whereas from line sampling, we get an estimate for the crown ratio as defined in Eq. 1.

Inserting the corresponding inclusion probabilities into Eq. 7 and Eq. 8, we get the following estimators for the total at a given sample location:

[9] $\hat{\tau}_{s}=\sum_{i=1}^{n_{s}} \frac{y_{i}}{\pi_{i}}=\frac{A}{L} \times \frac{h}{d} \times \sum_{i=1}^{n_{s}} \frac{\mathrm{H}_{\mathrm{i}}}{\mathrm{H}_{\mathrm{i}}}=E F_{L} \times n_{s}$

for line sampling and

[10] $\hat{\tau}_{s q, s}=\sum_{i=1}^{n_{s}} \frac{y_{i}^{2}}{\pi_{i}}=\frac{A}{\pi} \times\left(\frac{h}{d}\right)^{2} \times \sum_{i=1}^{n_{s}} \frac{H_{i}^{2}}{H_{i}^{2}}=E F_{P} \times n_{s}$

for point sampling. For point sampling the result is a sum of squares, while for line sampling a simple sum in non-transformed units is estimated. The terms left of the sum in Eq. 9 and Eq. 10 define an expansion factor, $E F$, equivalent to the basal area factor in horizontal point sampling, for expanding tree counts $n_{s}$ to per area values. In practical applications measurements would be limited to trees above a certain size threshold, so that the derived ratios are valid only for the set of trees fulfilling the threshold criterion. Finally, estimates from several sample locations are combined using Eq. 5 and Eq. 6, which in turn are inserted into Eq. 3 and Eq. 4 to provide an estimate of $r_{c}$ and $r_{c, \text { alt }}$. 


\section{Variance estimation}

Variance estimation for $\hat{r}_{c}$ is straightforward, while for $\hat{r}_{c, a l t}$ the situation is slightly more complicated. For the sake of completeness, we also provide variance estimators for the totals $\hat{\tau}$ and $\hat{\tau}_{s q}$, which, according to Gregoire and Valentine (2008, p 216), are:

[11] $\hat{v}(\hat{\tau})=\frac{1}{m(m-1)} \sum_{s=1}^{m}\left(\hat{\tau}_{s}-\hat{\tau}\right)^{2}$

And

[12] $\hat{v}\left(\hat{\tau}_{s q}\right)=\frac{1}{m(m-1)} \sum_{s=1}^{m}\left(\hat{\tau}_{s q, s}-\hat{\tau}_{s q}\right)^{2}$

As $\hat{r}_{c}$ is a non-linear function of the two variables $\hat{\tau}_{c}$ and $\hat{\tau}_{h}$, we apply Taylor linearization to approximate the variance of $\hat{r}_{c}$ (e.g. Särndal et al. 1992, p 179):

[13] $\hat{v}\left(\hat{r}_{\mathrm{c}}\right)=\frac{1}{\hat{\tau}_{h}^{2}} \times\left[\hat{v}\left(\hat{\tau}_{\mathrm{c}}\right)+\hat{r}_{c}^{2} \hat{v}\left(\hat{\tau}_{h}\right)-2 \hat{r}_{c} \hat{c}\left(\hat{\tau}_{\mathrm{c}}, \hat{\tau}_{h}\right)\right]$

where for $\hat{v}\left(\hat{\tau}_{\mathrm{c}}\right)$ and $\hat{v}\left(\hat{\tau}_{\mathrm{h}}\right)$ Eq. 11 is used and $\hat{c}\left(\hat{\tau}_{\mathrm{c}}, \hat{\tau}_{h}\right)$ is the covariance between the estimates for total crown length and total tree height:

$$
\hat{c}\left(\hat{\tau}_{c}, \hat{\tau}_{h}\right)=\frac{1}{m(m-1)} \sum_{s=1}^{m}\left(\hat{\tau}_{s, c}-\hat{\tau}_{c}\right)\left(\hat{\tau}_{s, h}-\hat{\tau}_{h}\right)
$$

For $\hat{r}_{c, \text { alt }}$, in addition to the ratio, we also have a square root transformation, which needs to be handled. Thus, $\hat{r}_{c, a l t}$ is squared to get $\hat{r}_{c, a l t, s q}$, for which the variance estimator is derived similar as in Eq. 13, by replacing $\hat{\tau}$ with $\hat{\tau}_{s q}$ and $\hat{r}_{c}$ with $\hat{r}_{c, a l t, s q}$.

To go from $\hat{v}\left(\hat{r}_{c, a l t, s q}\right)$ to $\hat{v}\left(\hat{r}_{c, a l t}\right)$, again Taylor approximation is applied. We approximate $\hat{r}_{c, a l t}$ as a function of $\hat{r}_{c, a l t, s q}$, i.e. $\hat{r}_{c, \text { alt }}=\sqrt{\hat{r}_{c, a l t, s q}}$ and use first order Taylor approximation to obtain:

[14] $\hat{v}\left(\hat{r}_{c, a l t}\right) \approx\left(\frac{0.5}{\hat{r}_{c, a l t}}\right)^{2} \times \hat{v}\left(\hat{r}_{c, a l t, s q}\right)$

We thus get $\hat{v}\left(\hat{r}_{c, a l t}\right)$ by applying a single factor to the known variance of $\hat{r}_{c, a l t, s q}$, and thus, $\hat{v}\left(\hat{r}_{c, a l t, s q}\right)$ is expanded if $\hat{r}_{c, a l t}<0.5$ and reduced if $\hat{r}_{c, \text { alt }}>0.5$.

Instead of presenting variances, we use the relative standard error, $s e_{r e l}$, in the Results section, which is calculated by dividing the square root of an estimator's variance by the estimator itself. For $\hat{r}_{c, a l t}$, applying Eq. 14, the relative standard error is interestingly exactly the half of that for $\hat{r}_{c, a l t, s q}$, i.e.

$s e_{r e l}=\frac{1}{\hat{r}_{c, a l t, s q}} \times 0.5 \times \sqrt{\hat{v}\left(\hat{r}_{c, a l t, s q}\right)}$.

\section{Study sites}

Sampling errors were studied in four Scots Pine dominated stands in the vicinity of the town Vindeln in northern Sweden. A summary is given in Tab. 1, based on basal area and height observations at the same 15 random locations that were used for point sampling. Basal area was measured with the traditional relascope
Table 1: Variables describing the four stands used for studying sampling error.

\begin{tabular}{lllll}
\hline & A & B & C & D \\
\hline Area (ha) & 35.7 & 34.9 & 33.9 & 16.7 \\
SI (height in m at 100 years) & 20 & 21 & 20 & 20 \\
Age (years) & 33 & 34 & 30 & 35 \\
Height (m) & 9.8 & 10.1 & 9.9 & 9.9 \\
Basal area (m2/ha) & 21.9 & 19.1 & 18.4 & 18.7 \\
\hline
\end{tabular}

method, using a basal area factor of 1 , and height was measured on a subjectively selected tree representing average basal area. Area, age and site index of the stands where taken from an available stand register. The additional measurements were made only in order to provide data for general stand descriptions. The average age of the stands was 33 years with a basal area weighted height of about $10 \mathrm{~m}$. Pine was the dominating tree species with shares of total basal area in the range from $83 \%$ to $92 \%$. The terrain in the four stands was rather flat not exhibiting more than $5 \%$ of slope, which could be handled well with the instrument prototype.

To study measurement errors, stands A and B (see Tab. 1) were used and an independent set of 11 sample locations was selected randomly. A description of the measured trees is given in Tab. 2.

\section{Instrument development}

The instrument was constructed of a thread rod serving as vertical basis, to which three metal markers covered with red shrink tubes for increased contrast were bolted. A wooden handle piece and a counting device complemented the tool. The distance to the eye of the observer was held constant and horizontal with a nonflexible fishing cord, which could slide freely along the rod, so that the instrument could be moved up and down while maintaining a fixed horizontal distance. The length between the two outer markers, which were used for aiming at tree height, was set to $40 \mathrm{~cm}$, while the two upper markers, used for aiming at the crown, were $20 \mathrm{~cm}$ apart. The distance to the eye was set to $30 \mathrm{~cm}$. The implication is that a hypothetical $10 \mathrm{~m}$ large tree would be included up to a distance of $7.5 \mathrm{~m}$. The vertical gauge constant (Brown and Mugasha 1988 ) is thus $4 / 3$ when selecting for height, defined by the ratio of the length between the markers, $h$, and the horizontal distance from the observer's eye, $d$. It was found that this ratio is at the lower limit from a field work point of view; for smaller ratios it becomes difficult to focus the two markers at the same time, influencing the judgment about whether a tree should be selected or not in a negative way. 


\section{Study on sampling error}

To study the statistical performance of the proposed method, for each stand and for each layout of sampling

Table 2: Summary of the data used for the measurement error study.

\begin{tabular}{lrrrrr}
\hline \multirow{2}{*}{ Satistic } & \multicolumn{1}{c}{ Dbh } & Height & \multicolumn{1}{c}{$\begin{array}{l}\text { Crown } \\
\text { length }\end{array}$} & $\begin{array}{c}\text { Crown } \\
\text { ratio }\end{array}$ & $\begin{array}{c}\text { Basal } \\
\text { area }\end{array}$ \\
\cline { 2 - 6 } & \multicolumn{1}{c}{$(\mathrm{cm})$} & \multicolumn{1}{c}{$(\mathrm{m})$} & \multicolumn{1}{c}{$(\mathrm{m})$} & & $\left(\mathrm{m}^{2}\right)$ \\
Mean & 14.8 & 9.5 & 5.9 & 0.6 & 21.1 \\
Min & 5.6 & 5.4 & 2.9 & 0.4 & 15.0 \\
Max & 24.7 & 12.3 & 10.2 & 1.0 & 26.0 \\
SD & 3.7 & 1.3 & 1.4 & 0.1 & 3.9 \\
\hline
\end{tabular}

Note: Dbh, diameter at breast height; SD, standard deviation

units (points or lines) 15 random locations were generated independent of each other. The selected sample locations were located with a hand-held GPS and trees were observed with the instrument. For point sampling the number of heights and crowns appearing larger than the respective opening angles were recorded while rotating $360^{\circ}$. For line sampling a $15 \mathrm{~m}$ long, north-south oriented sample line was used, with the sample location as starting point. Trees were observed at perpendicular distances to the eastern side of the line. On the trees no additional measurements had to be done.

Methods to handle issues related to boundary overlap were never applied as none of the sample units crossed a stand boundary. However, for the point sampling protocol the Walkthrough method as described in Ducey et al. (2004) should be applicable and for line sampling the Walkback method from Affleck et al. (2005). Alternatively, an external peripheral zone as suggested by Masuyama (1954) could be established around stands allowing sample locations to fall within the stand and the buffer zone but selecting objects of interest only within the stand.

Sampling errors related to total crown length, total tree height, and crown ratio - and the corresponding alternative definitions - were estimated using Eq. 11 to Eq. 14. To investigate the influence of sample size on the estimators, empirical population variances, $s^{2}$, were calculated multiplying the sampling errors with the actual sample size. Using the estimated population variances as if they were the true ones, variances for any given sample size could for example be estimated according to $\hat{v}\left(\hat{r}_{c}\right)=\frac{s_{r}^{2}}{m}$. The remaining estimators were treated in a similar way.

\section{Study on measurement errors}

Measurement errors are related to the selection of trees when applying the vertical relascope. There are two types of errors to account for in this context: commission and omission errors. A commission error is due to a faulty selection of a tree, i.e. a tree is selected by the observer although its physical parameters in combination with the sample point or line location imply that it should not have been included. On the opposite, an omission error occurs when a tree meets the physical conditions for selection but is missed by the surveyor during the inventory.

To assess the magnitude of omission and commission errors under real conditions, 11 sample locations were randomly selected in stands $\mathrm{A}$ and $\mathrm{B}$ and point sampling was applied, i.e. trees or crowns that appeared larger than the opening angle when rotating $360^{\circ}$ were selected. To control whether the actual selection was correct or not, the surrounding trees were carefully measured with regard to distance between observer and tree, tree height, and crown length, as defined in SLU (2011).

In total 254 trees were included in the relascope sample for the measurement error study; 193 were selected for height and 225 for crown length. Three different cases occur: (1) a tree is selected only because of its height, (2) a tree is selected only because of its crown length, and (3) a tree is selected both because of its height and its crown length. The last case was most abundant with 185 trees. To check for omission errors, 21 additional trees that appeared slightly smaller than the applied opening angle were carefully measured for height and crown length. From the data we have two sets of trees, one selected based on vertical relascope measurements and one control set, where selection was determined based on distance, height, and crown length measurements. The different error types were consequently assessed by comparing the two sets. One observer conducted all the relascope measurements.

\section{Comparison of $\boldsymbol{r}_{\boldsymbol{c}}$ and $\boldsymbol{r}_{\boldsymbol{c}, \boldsymbol{a l t}}$}

In this section we investigate under what conditions $r_{c}$ and $r_{c, \text { alt }}$ are equal and else how large the differences are expected to be, as it would be desirable to be able to apply both definitions and their related estimators interchangeably. In order to simplify the investigation somewhat, we square both definitions in the following instead of using the original ones: We thus examine when the following relationship holds:

[15] $r_{c}^{2}=r_{c, a l t}^{2} \Rightarrow \frac{\left(\sum y_{c, i}\right)^{2}}{\left(\sum y_{h, i}\right)^{2}}=\frac{\sum y_{c, i}^{2}}{\sum y_{h, i}^{2}}$

Now the entire population of $i=1,2, \ldots, N$ trees in a stand is regarded and $y_{c, i}$ and $y_{h, i}$ are used to represent crown length and tree height, respectively.

There exist two cases where perfect equality of Eq. 15 holds. They are (i) when crown length is a constant fraction of tree height for all trees in a stand and (ii) when the study variables have the same variance in relative terms. For the first case, by 
assuming a constant ratio $r_{c}=y_{c, i} / y_{h, i}$ for all population elements, one can easily show that

$$
r_{c, a l t}^{2}=\frac{\sum y_{c, i}^{2}}{\sum y_{h, i}^{2}}=\frac{\sum r_{c}^{2} y_{h, i}^{2}}{\sum y_{h, i}^{2}}=r_{c}^{2}
$$

Table 3: Number of trees selected in average per observation unit during the stand inventory. Results are separated after stand and inventory method with constant sample size for all groups.

\begin{tabular}{llrr}
\hline Stand & Method & No Heights & No Crowns \\
\hline A & Line & 20.3 & 26.7 \\
B & Line & 14.9 & 19.4 \\
C & Line & 12.3 & 16.1 \\
D & Line & 14.8 & 19.8 \\
A & Point & 27.9 & 43.3 \\
B & Point & 21.9 & 37.9 \\
C & Point & 20.6 & 33.2 \\
D & Point & 23.4 & 41.4 \\
\hline
\end{tabular}

So in a case of a constant ratio, $r_{c}^{2}=r_{c, \text { alt }}^{2}$, which is equivalent to $r_{c}=r_{c, a l t}$. This is rarely the case in natural stands but for even aged Pine plantations, as in our case, small variation among the $r_{i}$ might be expected and thus small differences between the two alternative crown ratio definitions.

For the second case we use the relationship

$$
\sum\left(y_{i}-\bar{y}\right)^{2}=\sum y_{i}^{2}-\frac{1}{N}\left(\sum y_{i}\right)^{2},
$$

where the squared sums as well as the sum of squares from Eq 15 are present, and consequently we rearrange for $\sum y_{i}^{2}$ resulting in:

$$
\text { [16] } \begin{aligned}
\sum y_{i}^{2} & =\frac{1}{N}\left(\sum y_{i}\right)^{2}+\sum\left(y_{i}-\bar{y}\right)^{2} \\
& =\frac{1}{N}\left(\sum y_{i}\right)^{2}+N \operatorname{Var}(y)
\end{aligned}
$$

From that we see that the difference between $\left(\sum y_{i}\right)^{2}$ and $\sum y_{i}^{2}$ depends on the empirical variance of the study variable. So, if the variance is known, the sum of heights and the sum of crown lengths could be calculated applying point sampling. The problem, however, is that the variation is unknown when trees are just counted with no further measurements done. A possibility could be to simply ignore the variation and accepting a deviation that depends on the variance of the study variables; if the variance is zero, there is no deviation. To show that $r_{c}$ and $r_{c, a l t}$ are the same if the two study variables, crown length and tree height, have the same relative variance, we define the relative variance as $\operatorname{Var}_{r e l}=\operatorname{Var}(y) / \bar{y}^{2}$. Rearranging for $\operatorname{Var}(y)$, we get $\operatorname{Var}(y)=\left(\sum y_{i}\right)^{2} \times \operatorname{Var}_{\text {rel }} \times N^{-2}$. Using this together with Eq 16 and replacing $\sum y_{i}^{2}$ in Eq 15, the following result can be obtained:

$$
\frac{\sum y_{c, i}^{2}}{\sum y_{h, i}^{2}}=\frac{N\left(\sum y_{c, i}\right)^{2}+N^{3} \operatorname{Var}_{c, r e l}\left(\sum y_{c, i}\right)^{2}}{N\left(\sum y_{h, i}\right)^{2}+N^{3} \operatorname{Var}_{h, r e l}\left(\sum y_{h, i}\right)^{2}}=\frac{\left(\sum y_{c, i}\right)^{2}}{\left(\sum y_{h, i}\right)^{2}}
$$

From that we see, that if $\operatorname{Var}_{c, r e l}=\operatorname{Var}_{h, r e l}, r_{c}$ equals $r_{c, a l t}$.

To study the magnitude of the difference between $r_{c}$ and $r_{c, a l t}$ when the special cases described do not hold, a small simulation study was made. Two sets of random numbers were generated from a normal distribution corresponding to heights and crown lengths, respectively; with means and standard deviations taken from the measurement error data, i.e. from the part of this study where all tree heights and crown lengths were measured. From the two sets $r_{c}$ and $r_{c, a l t}$ were calculated. The procedure was finally repeated 1000 times using the generated observations to calculate the average difference between the two ratios

[17] Diff $=\frac{\sum\left(r_{c}-r_{c, a l t}\right)}{k}$

and the standard error from a linear regression between $r_{c, a l t}$ and $r_{c}$

[18] $\operatorname{StdErr}=\sqrt{\frac{\sum\left(r_{c}-r_{c, p r e d}\right)^{2}}{k-2}}$

where $k$ denotes the number of repetitions and $r_{c, \text { pred }}$ the predicted values for $r_{c}$ from $a+b \times r_{c \text {,alt }}$ with $a$ and $b$ being the regression coefficients.

The simulation itself was then repeated in a way that the variation of the two data sets (heights and crown lengths) was changed in terms of the coefficient of variation from 0 to 0.25 in steps of 0.01 . The mean and the size of the individual sets were kept constant.

\section{Results}

\section{Sampling error}

The average number of trees per observation unit selected during the inventory is reported in Tab. 3 separated by stands, inventory method, and variables observed. When observing heights in average across all stands 15.6 trees were selected with lines and 23.5 trees with points. For crowns the numbers were 20.5 and 39, respectively. Two general observations can be made: (1) with the sample line length used in average fewer trees were selected from sample lines than from sample points; and (2) consistently more trees were included due to their crowns than due to their heights.

Estimates of $r_{c}$ range from $65 \%$ to $67 \%$, while that of $r_{c, a l t}$ lie between $62 \%$ and $67 \%$; all in all showing only little variation. Relative standard errors of $\hat{r}_{c}$ and $\hat{r}_{c, \text { alt }}$ and for the related totals, $\hat{\tau}$ and $\hat{\tau}_{s q}$, from which they are derived, are given in Fig. 3. The underlying variances used to calculate the relative standard errors were estimated using Eq. 11 (line sampling) and Eq. 12 
(point sampling) for the totals, and Eq. 13 (line sampling) and Eq. 14 (point sampling) for the ratios. The values at a sample size of 15 give the actual error estimates from the inventory of the single stands and variables. In general sample errors can be considered as low and they show the typical decrease with increasing sample size. When looking at the four

Table 4. Number of commission (Com) and omission (Om) errors in relation to the number of trees selected by the vertical relascope and by control measurements, respectively.

\begin{tabular}{|c|c|c|c|c|c|c|}
\hline \multirow[t]{2}{*}{ Variable } & \multicolumn{2}{|c|}{ Selection based on } & \multicolumn{2}{|c|}{$\begin{array}{l}\text { No. of } \\
\text { errors }\end{array}$} & \multicolumn{2}{|c|}{$\begin{array}{l}\text { Error } \\
\text { percentage }\end{array}$} \\
\hline & Relascope & Control & Com & $\mathrm{Om}$ & Com & Om \\
\hline Height & 193 & 200 & 7 & 14 & 3.6 & 7.0 \\
\hline Crown & 225 & 242 & 2 & 19 & 0.9 & 7.9 \\
\hline
\end{tabular}

stands, the average differences of the error curves between point and line sampling are marginal, whereas the spread between the curves seems to be slightly larger for point sampling. The average relative standard error at sample size 15 for either point or line sampling is $7.1 \%$ and $6.9 \%$ for tree heights, $5.4 \%$ and $5.9 \%$ for crown lengths, and $2.1 \%$ and $2.7 \%$ for the two crown ratios. The remarkably low sampling errors for the two crown ratio estimates are due to the strong correlation between sum of heights and sum of crown lengths estimates (or their corresponding squares), which reduces the variance estimates (see Eq. 13) accordingly. For the four stands the correlations in the line based estimates ranged from 0.86 to 0.96 .

Time consumption per sample location was measured for stand D after the observer got used to the method; in average 5.8 minutes were needed for lines and 5.1 minutes for points.

\section{Measurement errors}

In Tab. 4 the results of the study on measurement errors are summarised. We present the number of trees selected either for their height or their crown length and the corresponding number of errors made. Relascope selection means that only such trees that have been chosen by the observer using the vertical relascope are included. Control measurement selection, includes all trees based on actual measurements of distance, height, and crown length, and not on visual selection with the vertical relascope. A distinction is made between commission and omission errors.

Fewer trees were selected with the vertical relascope than should have been included based on the control measurements. This was less pronounced for heights than for crowns as commission and omission errors level off to some extent. For crowns only few commission errors were observed, so the underestimation of totals, as estimated from Eq. 5 and
Eq. 6, is stronger here. The general outcome is that it is more likely to oversee a tree than to include too many.

In Fig. 4 the effect of observation distance on the occurrence of errors is illustrated. We present the frequency distribution of all trees measured except the additional measured trees that showed no errors, and, extracted from that, the related errors made for either height or crown selection, where omission and commission errors are combined. In the present case the majority of trees are situated around $5 \mathrm{~m}$ from the observation point and the distribution itself has a bellshaped form. The maximum distance observed was $11.32 \mathrm{~m}$. Errors did not occur in the three smallest distance classes, while in all other classes at least one error was observed. In absolute numbers most errors were committed between $5 \mathrm{~m}$ and $8 \mathrm{~m}$, on the one hand due to a higher number of trees and on the other hand due to a higher distance and decreasing visibility. In relative terms, the error rates increased substantially with distance. The two largest distance classes did not contain any height errors, as neither a tree was selected for height there, nor was one overseen. The occurrence of errors in combination with variables related to tree size showed no effects.

\section{Comparison of $\mathrm{r}_{\mathrm{c}}$ and $\mathrm{r}_{\mathrm{c} \text {,alt }}$}

The results of the simulation study are given in Fig. 5, where the average difference and the standard error as defined in Eq. 17 and Eq. 18 are given in relative terms with respect to $r_{c}$. Variability in the two sets of observations, i.e. tree height and crown length, is described in terms of the coefficient of variation (CV). From the figure we see that with increasing variation, the standard error increases as well, so that a prediction of $r_{c}$ from $r_{c, \text { alt }}$ is less accurate. However, in contrast to that, the average difference between $r_{c}$ and $r_{c, \text { alt }}$ can be zero, even with high variation, if the relative variances of tree heights and crown lengths are the same, as shown before theoretically. Otherwise, we observe an increasing average difference. When there is no variation at all, $r_{c}$ and $r_{c, a l t}$ are equal to each other. In general, deviations between $r_{c}$ and $r_{c, \text { alt }}$ were small, even for high levels of variations in the study variables. The maximum value for the average difference is $3 \%$ of $r_{c}$ and that for the standard error $1.4 \%$ of $r_{c}$, respectively.

\section{Discussion}

We showed that with the proposed method crown ratios for tree populations can be estimated with high precision at low time consumption. For both line- and point sampling, relative standard errors of less than $10 \%$ could be achieved with just five sample locations due to ratio estimation in combination with the two highly correlated variables total tree height and totals 
crown length or their corresponding sum of squares. However, it should be noted that we applied the method to planted, even-aged stands with quite homogenous conditions; other conditions might require larger sample sizes. Despite of that, an inclusion of crown ratio estimation into existing timber surveys can be considered straightforward, giving forest managers the possibility for an objective assessment of the crown ratio in a stand. If the crown ratio falls below a certain threshold, e.g. $50 \%$ of tree height, this may indicate a need for thinning. The decision about thinning can thus be based on information that is directly linked to the growth of trees (Dean and Baldwin 1996). In addition to the assessment of thinning needs, the method might be applicable for a monitoring of past thinning activities in the course of regularly repeating inventories such as NFIs.

An alternative approach to crown ratio estimation would be to use fixed area plots in combination with measurements of individual tree heights and crown lengths. An estimate of the crown ratio would then be formed in a similar way as given in Eq. 3 from estimates of total crown length and tree height. In contrast to the relascope method, inclusion probabilities do not depend on tree characteristics and are constant once a plot size is chosen. Thus, sampling precision can be expected to be higher for relascope sampling at a given sample size, as the study variables are proportional to the inclusion probabilities (Gregoire and Valentine 2008, pp 256-257). Further on, relascope sampling would also be preferable with respect to time consumption when interest is merely in crown ratio estimation. On the other hand, fixed area plots might have an advantage in situations of estimating change. For relascope sampling, a higher variation between different surveyors can be expected, what might hide real trends (Ringvall and Ståhl 1999). For crown ratio estimation, however, experiences are lacking and more research is needed into that direction.

Yet another alternative would be to use traditional horizontal point sampling for crown ratio estimation, where a standard relascope is used to select trees based on their basal area. Larger trees thereby get a higher probability of inclusion than smaller ones and thus this method could be expected to be slightly more efficient than fixed area plots as thick trees tend to be higher and have longer crowns than thin trees.

The study on measurement errors when using the vertical relascope revealed a slight underestimation, i.e. the number of wrongly selected trees was lower than the number of missed trees and thus the two error types did not level off. As the effect was stronger when selecting crowns, this also influenced the ratio estimate, which turned out to be $3.5 \%$ to $4 \%$ lower compared to the ratio from correctly selected trees. These results, however, are based on measurements by one single surveyor, for more general results a larger study would be needed. However, the observed error rates are in accordance with the ones reported in other studies on relascope measurements (e.g. Danielsson and Larsson 1964; Ringvall and Ståhl 1999).

Constraints and weaknesses of the method are geometrical issues when the vertical axes of tree and instrument are not parallel, the difficulty to apply the method when sighting conditions are poor, and the difference between $r_{c}$ and $r_{c \text {,alt }}$ in case point sampling is applied. The first issue is illustrated in Fig. 6 showing several cases of sighting a tree. Under a) and b) the instrument is held parallel to the tree, which assures correct selections. Under c) and d) a tree is leaning towards or away from the observer, while the instrument is held vertical. Opposite cases, upright standing trees observed through a tilted instrument, are illustrated under e) and f). For cases where the vertical axes of the instrument and the trees are not parallel, the decision whether to select a tree or not may be biased. For c) and e) fewer trees can be expected whereas the opposite holds for d) and f). In our case with straight and upright trees, this was a rather small issue but under other conditions this effect might lead to some bias, especially as it is difficult for an observer to judge whether a tree is leaning towards or away from the point of observation.

In contrast to basal area estimation, sighting conditions play a crucial role as diffuse tree crowns tend to overlap much more than rather sharply separated stems. It was often quite difficult to separate the crowns from each other and to aim at the correct tree tops. Another issue is that the beginning of the green crown can be difficult to locate from a distance, especially when the lower branches are only sparsely needled. The effect could be that crown lengths estimated with the vertical relascope are somewhat shorter than they would be if measured at the tree.

The issue of the difference between $r_{c}$ and $r_{c, \text { alt }}$ could be solved as presented in the method and results section. The simulation study indicated that either point or line sampling would give similar results provided that (i) the ratio between crown length and tree height is constant for all trees; (ii) both study variables have equal variation in relative terms; or (iii) the empirical variance of the study variables is rather small or zero. The outcome very much depends on the population at hand and it is necessary to have some idea about the variation, which might be obtained from additional measurements or earlier studies. In our study $r_{c}$ was equal to 0.66 on average across all four stands, while the corresponding value for $r_{c \text {,alt }}$ was 0.65 .

However, when choosing between point and line sampling we recommend line sampling, which apart from providing easily interpreted measures has other advantages. One is the possibility of an easier 
adjustment of the average number of trees that are selected per sample unit. With points the only way to influence this is to change the opening angle, which only works within certain limits. If the angle is too narrow even trees far away would be included, and such trees are easily overseen. A wider opening angle, on the other hand, makes it difficult to sight at a tree as the two markers are too far away from each other to focus them at the same time. With line sampling one could select an opening angle that is easy to use in the field and adjust the number of trees per unit simply by adjusting the line length. A further advantage of line sampling is the improved visibility because the observer is allowed to move along the line by design.

In our study we used a vertical gauge constant of $4 / 3$ for measuring tree heights and $2 / 3$ for measuring crowns. Bickerstaff (1961) and Hitchcock (1979) used a constant of one, which we initially tried with the conclusion that it was too narrow in our conditions, and thus led to the inclusion of too many trees (about 40), when aiming for height. Other authors, e.g. Brown and Mugasha (1988) and Mugasha (1989), used a gauge constant of two, i.e. height is twice the critical distance. With that angle our judgement was that the selection of trees was insecure, as the two markers could not be focused at the same time.

Another issue is that consistently more trees were selected when aiming at crowns than at heights. The chosen opening angle for selecting crowns was half as large as the one for heights, whereas, in reality the crown ratio was around $60 \%$ in the case study stands.

In conclusion, the presented way of applying the relascope principle to crown ratio estimation is, to our best knowledge novel in the sense that it has not been used before for estimating crown ratios of forest stands. Under the reported conditions the method proved to work well both practically and statistically.

\section{Aknowledgements}

We thank the two anonymous reviewers for their valuable comments that helped to improve the quality of the manuscript.

\section{References}

Agestam, E. 2009. Skogskötselserien - Gallring, Skogsstyrelsen [online]. Available from: www.skogsstyrelsen.se/skogsskotselserien [Accessed 22 February 2012].

Affleck, D.L.R., Gregoire, T.G., and Valentine, H.T. 2005. Edge effects in line intersect sampling with segmented transects. Journal of Agricultural, Biological, and Environmental Statistics 10(4): 460-477. doi: 10.1198/108571105X81355.

Beers, T. 1974. Vertical line sampling for regeneration surveys. In Proceedings of a workshop on inventory design and analysis, Fort Collins, CO, 1974. Edited by W. Frayer, G. Hartman, and D. Bower. Society of American Foresters, Washington, D.C. pp. 246-260.

Bickerstaff, A. 1961. A variable quadrat regeneration survey method. The Forestry Chronicle 37(1): 39-54.

Bitterlich, W. 1948. Die Winkelzählprobe. Allgemeine Forstund Holzwirtschaftliche Zeitung 59(1/2): 4-5.

Bitterlich, W., 1984. The relascope idea: relative measurements in forestry. Commonwealth Agricultural Bureaux, Farnham Royal, Slough, UK.

Brown, K.M., and Mugasha, A.G. 1988. A two-stage method for horizontal point sampling in young forest stands. Canadian Journal of Forest Research 18(10): 1340-1343. doi :10.1139/x88-207.

Cochran, W.G., 1977. Sampling Techniques. Third Edition. John Wiley \& Sons, New York.

Danielsson, I., Larsson, U., 1964. Personal error in relascope measurements. Skogen, 51(3): 90-95.

Dean, T.J., Baldwin Jr., V.C. 1996. Growth in loblolly pine plantations as a function of stand density and canopy properties. Forest Ecology and Management, 82(1-3): 4958. doi: 10.1016/0378-1127(95)03694-6

Ducey, M., Gove, J., Valentine, H. 2004. A walkthrough solution to the boundary overlap problem. Forest Science 50(4): 427-435.

Ducey, M.J., Kershaw, J.A. 2011. Vertical point sampling with a camera. Northern Journal of Applied Forestry 28(2): 61-65.

Gove, J., Williams, M., Ståhl, G., Ducey, M. 2005. Critical point relascope sampling for unbiased volume estimation of downed coarse woody debris. Forestry 78(4): 417-431.

Gove, J.H., Ringvall, A., Ståhl, G., Ducey, M.J. 1999. Point relascope sampling of downed coarse woody debris. Canadian Journal of Forest Research 29(11): 1718-1726. doi: 10.1139/x99-119.

Gregoire, T.G., Valentine, H.T. 2008. Sampling Startegies for Natural Resources and the Environment. Applied Environmental Statistics, Chapman \& Hall/CRC, Boca Raton.

Grosenbaugh, L.R. 1958. Point-Sampling and Line-Sampling Probability Theory, Geometric Implications, Synthesis Occasional Paper 160. USDA Forest Service, Southern Forest Experiment Station.

Hasenauer, H., Monserud, R.A. 1996. A crown ratio model for Austrian forests. Forest Ecology and Management 84(1-3): 49-60.

Hirata, T., 1955. Height estimation through Bitterlich's method - vertical angle-count sampling. Journal of the Japanese Forestry Society 37(11): 479-480.

Hitchcock, H.C. 1979. Biomass of southern hardwood regeneration estimated by vertical line sampling. Journal of Forestry 77(8): 474-477.

Horvitz, D.G., Thompson, D.J. 1952. A generalization of sampling without replacement from a finite universe. Journal of the American Statistical Association 47(260): 663-685.

Kitamura, M. 1962. On an estimate of the volume of trees in a stand by the sum of critical heights. Kai Nichi Rin Ko 73: 64-67.

Lappi, J., Smolander, H., Kotisaari, A. 1983. Height relascope for regeneration surveys. Silva Fennica 17(1): $77-82$. 
Leites, L.P., Robinson, A.P., Crookston, N.L. 2009. Accuracy and equivalence testing of crown ratio models and assessment of their impact on diameter growth and basal area increment predictions of two variants of the Forest Vegetation Simulator. Canadian Journal of Forest Research, 39(3): 655-665. doi: 10.1139/X08-205.

Lessard, V.C., McRoberts, R.E., Holdaway, M.R. 2001. Diameter growth models using Minnesota forest inventory and analysis data. Forest Science, 47(3): 301310.

Lynch, T.B. 1990. Stand volume estimation from tree counts in the context of vertical line sampling. Canadian Journal of Forest Research 20(3): 274-279. doi: 10.1139/x90-040.

Masuyama, M. 1954. On the error in crop cutting experiment due to the bias on the border of grid. Sankhyā 14(3): 181186

McTague, J.P. 2010. New and composite point sampling estimates. Canadian Journal of Forest Research 40(11): 2234-2242.

Mugasha, A.G. 1989. Evaluation of simple competition indices for the prediction of volume increment of young jack pine and trembling aspen trees. Forest Ecology and Management 26(3): 227-235. doi: 10.1016/03781127(89)90123-0.

Ringvall, A., Ståhl, G. 1999. On the Field Performance of Transect Relascope Sampling for Assessing Downed
Coarse Woody Debris. Scandinavian Journal of Forest Research, 14(6): 552-557. doi: 10.1080/02827589908540820.

Särndal, C.E., Swensson, B., Wretman, J. 1992. Model Assisted Survey Sampling. Springer, New York.

SLU 2011. Fältinstruktion 2011. Riksinventeringen av Skog. Institutionen för Skoglig Resurshushållning, Institutionen för Mark och Miljö, Umeå, Uppsala.

Stenberg, P., Korhonen, L., Rautiainen, M. 2008. A relascope for measuring canopy cover. Canadian Journal of Forest Research 38(9): 2545-2550. doi: 10.1139/X08-081.

Ståhl, G. 1998. Transect relascope Sampling-A method for the quantification of coarse woody debris. Forest science 44(1): 58-63.

Ståhl, G., Gove, J., Williams, M., Ducey, M. 2010. Critical length sampling: A method to estimate the volume of downed coarse woody debris. European Journal of Forest Research 129(6): 993-1000. doi: 10.1007/s10342-0100382-3.

Strand, L. 1957. "Relaskopisk" høde- og kubikmassebestemmelse. Norsk Skogbruk 3: 535-538.

Tobin, B., Black, K., Osborne, B., Reidy, B., Bolger, T., and Nieuwenhuis, M. 2006. Assessment of allometric algorithms for estimating leaf biomass, leaf area index and litter fall in different-aged Sitka spruce forests. Forestry(4): 79, 453-465. doi: 10.1093/forestry/cpl030. 
Figure 1. Inclusion zones when applying the vertical relascope combined with either point or line sampling. The white dots are the sample locations, the dark grey dots the trees, and the grey shaded areas the corresponding inclusion zones.

a)

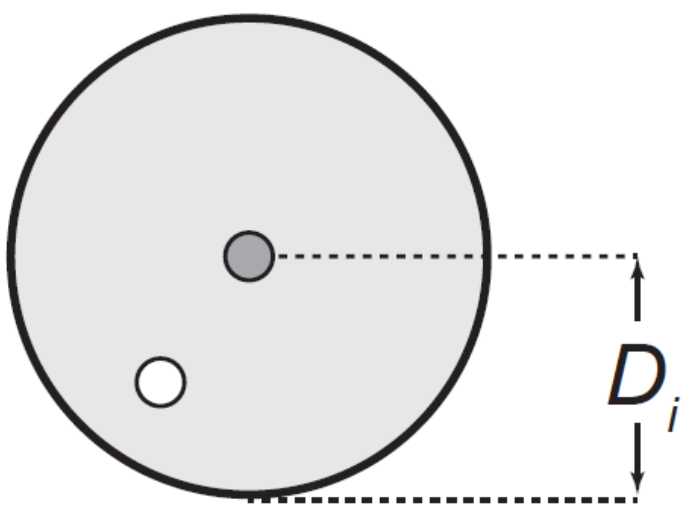

b)

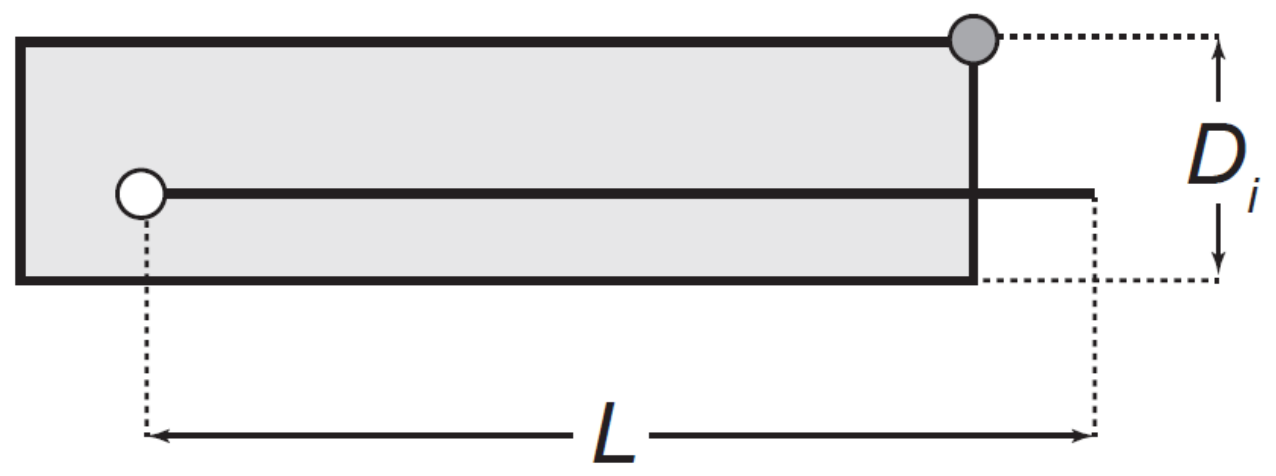


Figure 2. Illustration of the theoretical concept when using the vertical relascope: a) selection of tree height; b) selection of crown length; c) selection of crown length in sloped terrain with observer position above the tree top; and d) selection of crown length in sloped terrain with observer position below the base of a tree.

a)

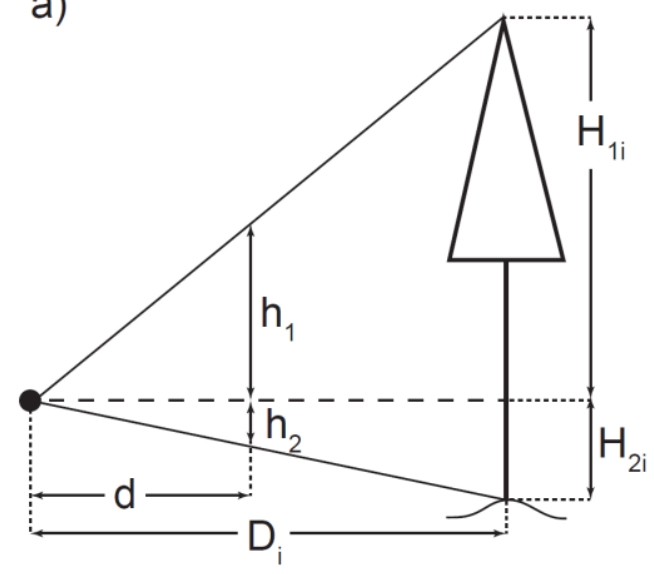

b)

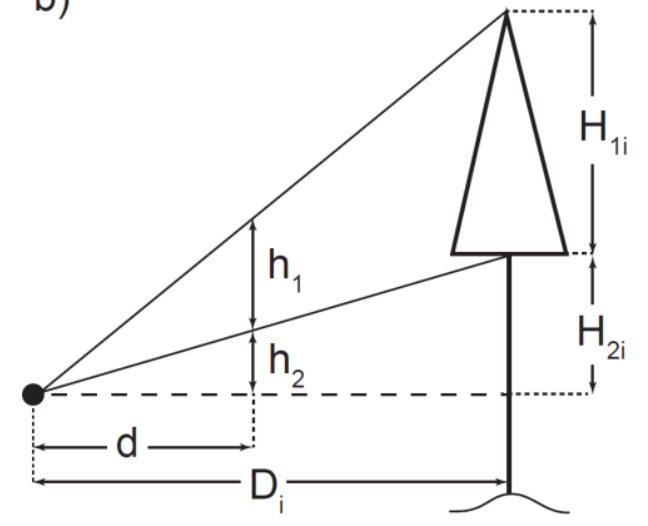

c)

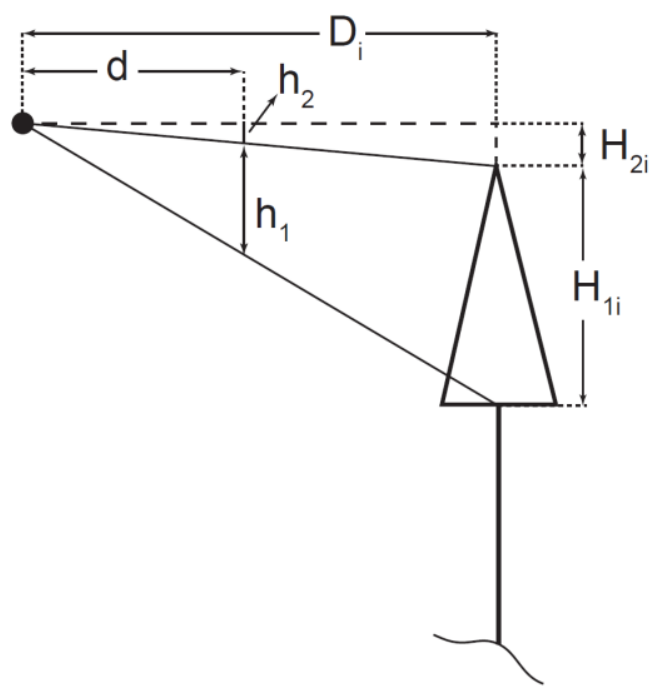

d)

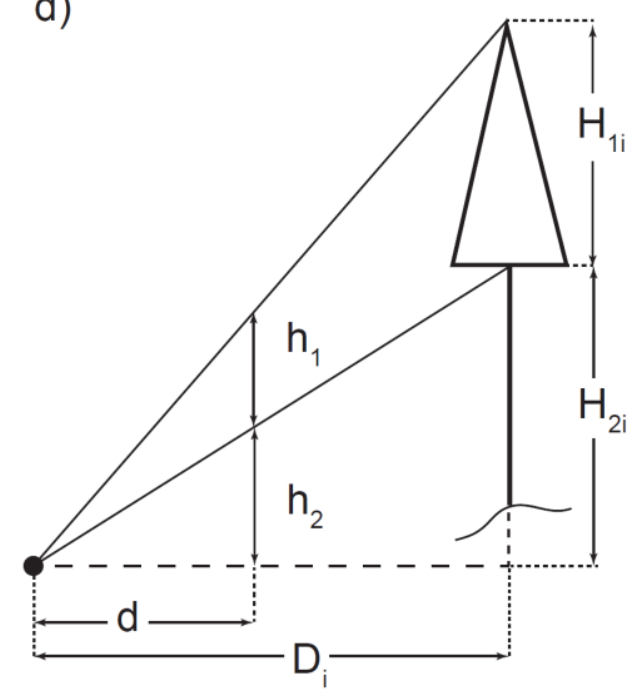


Figure 3. Change of sampling error (expressed as standard error in relation to the mean) with increasing sample size for the four stands inventoried; separated by the plot design (point and line sampling) and the variable observed (height, crown length, and crown ratio).

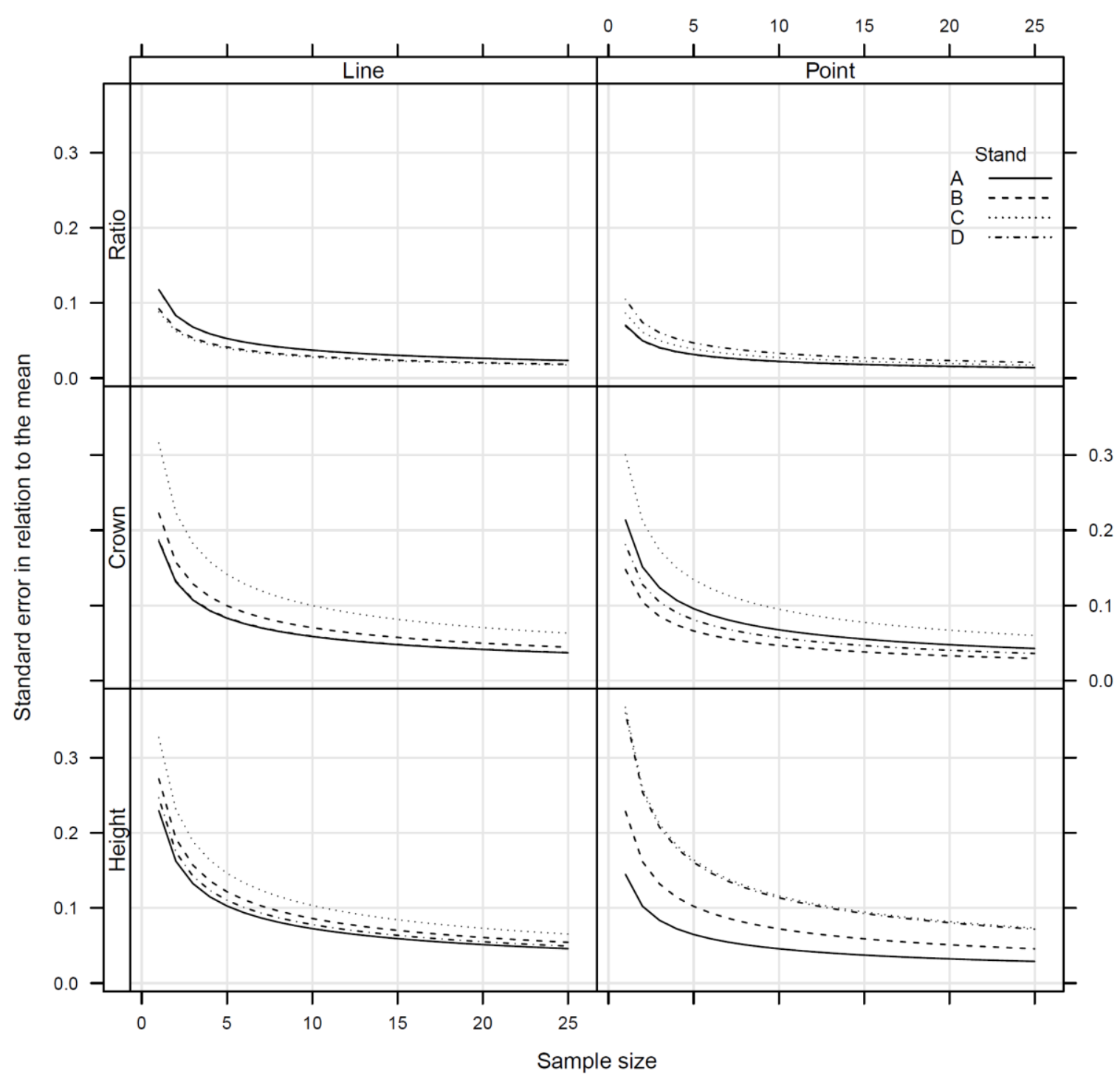


Figure 4. Frequency distribution of observation distances for all trees measured (white) and for trees with selection errors when either height (black) or crown length (grey) was measured.

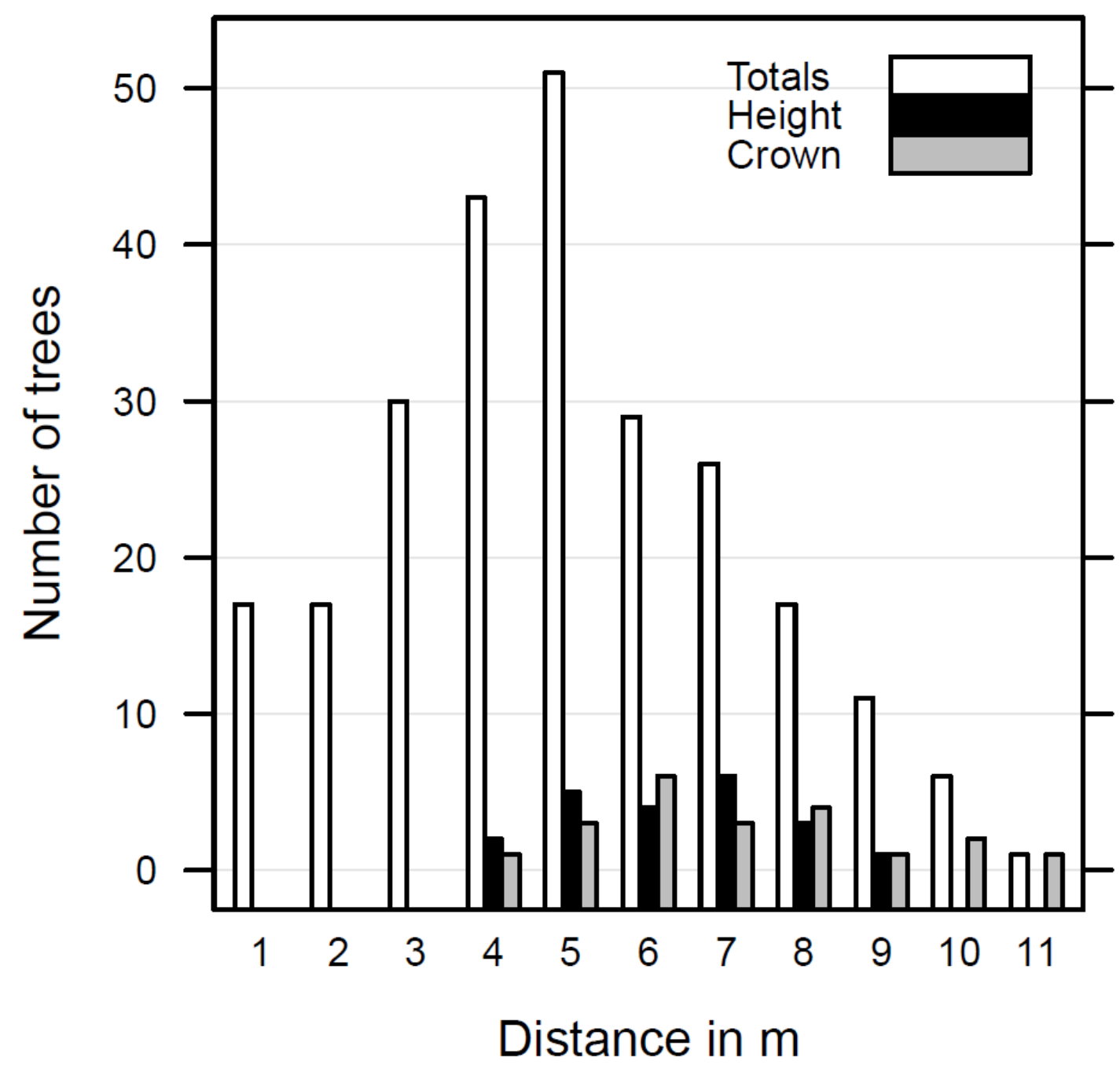


Figure 5. Average difference (Eq. 17) and standard error (Eq. 18) between $r_{c}$ and $r_{c \text {,alt }}$; related to $r_{c}$ and in dependence of the variation in tree height and crown length. Results were obtained from a simulations study with 1000 repetitions.
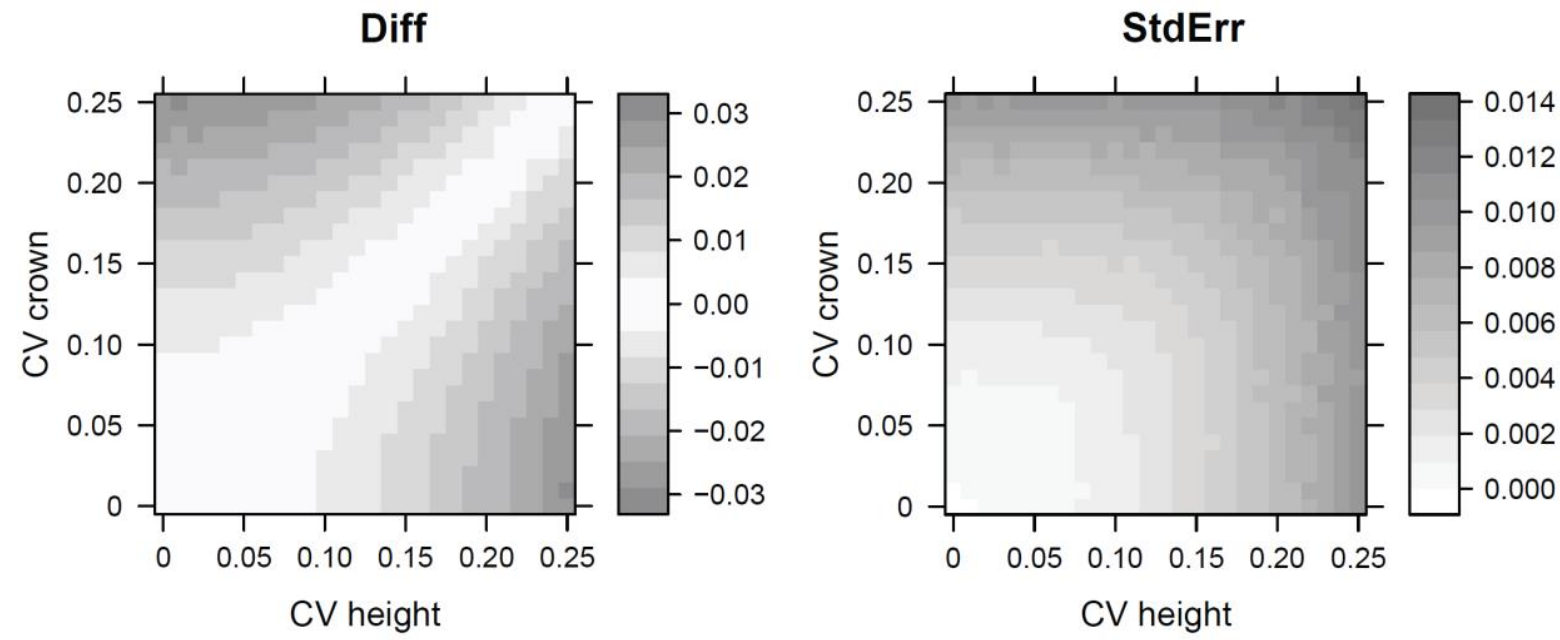
Figure 6. Typical situations when applying the vertical relascope: under a) and b) trees are selected correctly. For the other cases trees and the instrument are not vertically aligned leading to either overestimation ( $\mathrm{d}$ and $\mathrm{f}$ ) or to underestimation (c and e).

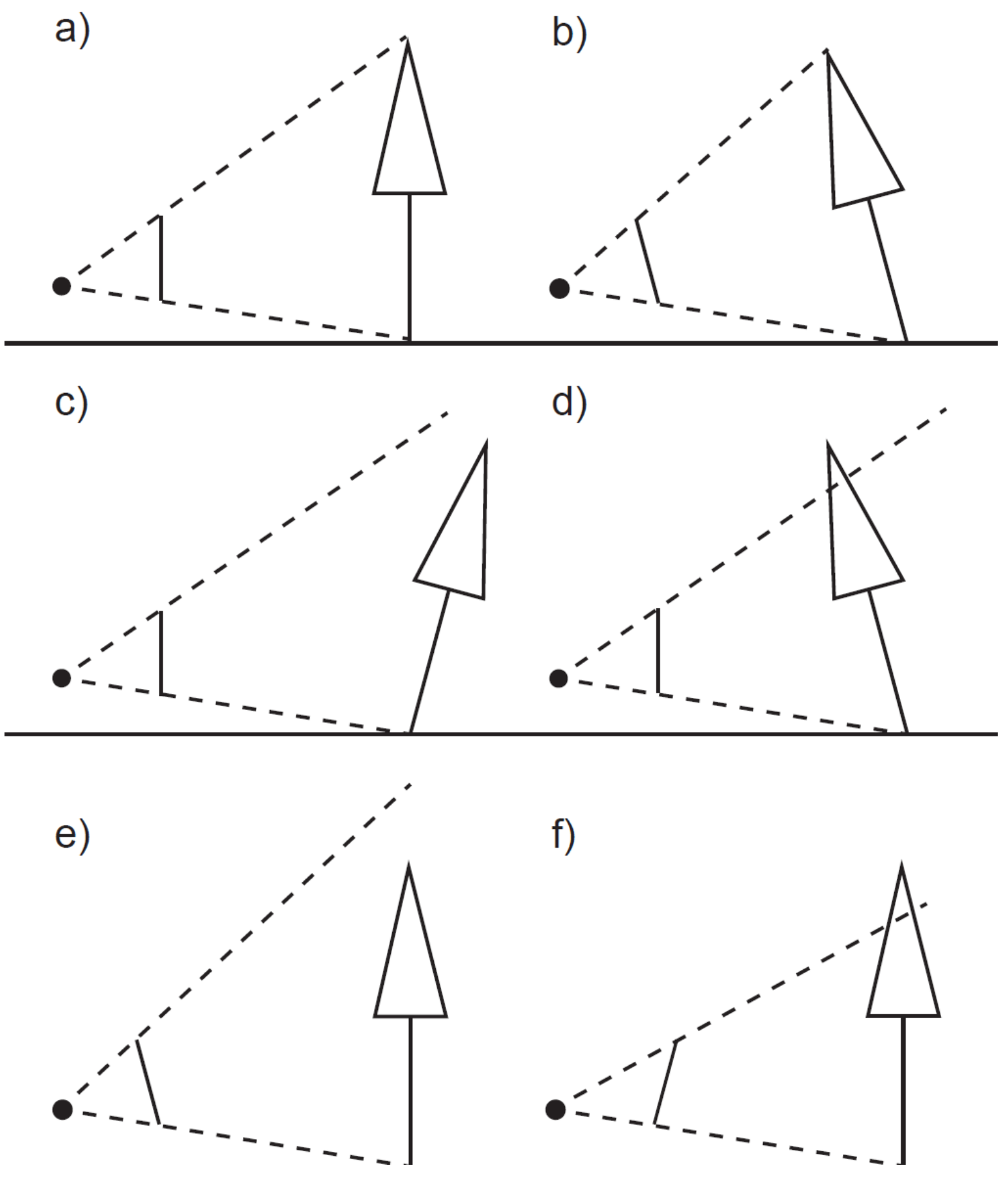

